\title{
Random gauge field effects on the conductivity of graphene sheets with disordered ripples
}

\author{
Rhonald Burgos, Jesus Warnes, Leandro R. F. Lima, and Caio Lewenkopf \\ Instituto de Física, Universidade Federal Fluminense, 24210-346 Niterói RJ, Brazil
}

(Dated: March 8, 2022)

\begin{abstract}
We study the effect of disordered ripples on the conductivity of monolayer graphene flakes. We calculate the relaxation times and the Boltzmann conductivities associated with two mechanisms. First, we study the conductivity correction due to an external in-plane magnetic field $B_{\|}$. Due to the irregular local curvature found at graphene sheets deposited over a substrate, $B_{\|}$can be mapped into an effective random magnetic field perpendicular to the graphene surface. Second, we study the electron momentum relaxation due to intrinsic pseudo magnetic fields originated from deformations and strain. We find that the competition between these mechanisms gives rise to a strong anisotropy in the conductivity tensor. This result provides a new strategy to quantitatively infer the strength of pseudo-magnetic fields in rippled graphene flakes.

PACS numbers: 72.80.Vp,73.23.-b,72.10.-d
\end{abstract}

\section{INTRODUCTION}

The electronic transport properties of bulk graphene are quite remarkable ${ }^{1-4}$. At room temperature graphene has mobilities ${ }^{5}$ as high as $\mu \approx 15,000 \mathrm{~cm}^{2} \mathrm{~V}^{-1} \mathrm{~s}^{-1}$, that are significantly larger than any other semiconductor. At low temperature, however, the typical mobilities increase only up to $\mu \approx 200,000 \mathrm{~cm}^{2} \mathrm{~V}^{-1} \mathrm{~s}^{-1}$, which is a disappointing figure as compared with high quality GaAs heterostructures. These observations triggered an intense theoretical and experimental activity to understand the disorder mechanisms that limit the mobility in graphene (see, for instance, Refs. 1-4 for a review). The theoretical understanding, acquired from the analysis of the Boltzmann equation ${ }^{1,3}$, numerical simulations ${ }^{2,6,7}$, and field theoretical techniques ${ }^{8}$ is that extrinsic disorder such as ad-atoms absorbed in the graphene surface, substrate charge inhomogeneities, and intrinsic disorder such as vacancies play a key role.

Motivated by recent experiments ${ }^{9-12}$, we study the effect of extrinsic and intrinsic random gauge potential disorder due to strain. We show that, although unlikely to be dominant in graphene deposited over standard substrates, these kinds of disorder give unique and sizable contributions to the conductivity. For high-quality substrates, there are even experimental evidences ${ }^{12}$ that random strain can be a good candidate for the leading electron relaxation mechanism in on-substrate graphene.

Standard electronic transport experiments use samples where graphene flakes are deposited over an insulating substrate. In such setting, it has been experimentally established that the graphene surface is characterized by disordered static ripples ${ }^{13-16}$. For $\mathrm{SiO}_{2}$ substrates, the latter have typical lengths of $\lambda \approx 5-30 \mathrm{~nm}$ and characteristic heights of $h_{\mathrm{rms}} \approx 0.2-0.5 \mathrm{~nm}$.

Lattice deformations due to ripples change the distance between the atoms in the graphene sheet. At the quantum level, site lattice displacements change the orbital bonding between the corresponding atoms, modifying the electronic structure of the material. In graphene, whose low-energy electronic properties are nicely described by a nearest-neighbor tight-binding model ${ }^{1}$, the occurrence of ripples change the tight-binding hopping terms ${ }^{1,17,18}$. For distortions with length scales much larger than the lattice parameter, characteristic to most samples ${ }^{13-16}$, it has been shown that the tight-binding model can be mapped into an effective Dirac Hamiltonian with a pseudo-magnetic vector potential ${ }^{1,19-21}$ that depends on the lattice distortions. Hence, random ripples give rise to an intrinsic random gauge potential. The experimental evidences of pseudo-magnetic fields are scarce and indirect, but quite remarkable. Strain fields have been invoked to associate the local density of states observed in graphene nanobubbles ${ }^{22}$ to Landau levels with energies corresponding to very high magnetic fields. To the best of our knowledge, no transport experiment has yet observed manifestations of this physical picture.

Random magnetic fields can also be achieved by realizing that disordered ripples in graphene and the roughness at semiconductor interface heterostructures share several common features. Starting at the late 80 's, a number of ingenious methods where used to characterize the interface roughness in the heterostructures ${ }^{23-25}$. One idea is particularly suited for graphene studies: By applying a strong magnetic field $B_{\|}$, aligned to the plane of a heterostructure interface confining the two-dimensional electron gas (2DEG), the (smooth) interface roughness disorder gives origin to a local random magnetic field perpendicular to the 2DEG surface. The analysis of the electronic transport properties as a function of the applied magnetic field gives quantitative information about the interface roughness.

This setting was nicely explored by a recent experiment ${ }^{9}$, that combined information of the average conductivity and its weak localization correction ${ }^{26,27}$ in graphene to extract the sample characteristic $\lambda$ and $h_{\text {rms }}$. This procedure have been also used in the experimental study of other graphene systems ${ }^{10,11}$. Theory ${ }^{28}$ shows that an applied $B_{\|}$on a rough surface gives rise to an effective dephasing $\ell_{\phi}$ and to the suppression of the weak 
localization peak. In addition to this quantum correction, the random magnetic field due to $B_{\|}$also contributes to the electron momentum relaxation, which at high doping is accounted for by the Boltzmann theory ${ }^{9}$. This nice analysis does not consider the effect of intrinsic pseudomagnetic fields due to strain, discussed above.

Our focus is different. We study the combined effect of intrinsic and extrinsic random magnetic fields in the Drude conductivity. We revisit the analysis of the Boltzmann equation in graphene $e^{1,2,4,29}$ and calculate the contributions of random magnetic fields to the Drude conductivity. We show that the conductivity corrections due to an applied in-plane magnetic field $B_{\|}$on a rippled graphene flake depend on the direction of $B_{\|}$and are very anisotropic. We find that this result can be reconciled with the experimental findings of Ref. 9 by theoretically treating the effect of strain and $B_{\|}$at the same footing. We also show that the combined effect of both sources of random magnetic fields provides a new experimental path to quantitatively probe the effects of strain fields in the low-energy electronic dynamics of graphene.

The paper is structured as follows: In Section II we present the disorder model we employ to describe ripples and the corresponding effective Hamiltonian. We briefly explain the origin of an effective random field due to an applied in-plane magnetic field $B_{\|}$, as well as from the intrinsic strain field due to ripples. In Sec. III we calculate the contributions to the Drude conductivity due to $B_{\|}$and strain using the Boltzmann transport equation. We show how to account for the disorder potential anisotropy and discuss its consequences comparing with experiments. Finally, in Sec. IV we present our conclusions and an outlook.

\section{MODEL HAMILTONIAN}

In this section we present a model to study the effect of extrinsic and intrinsic sources of a random magnetic fields in the dynamics of electrons in corrugated graphene monolayer samples.

Close to the charge neutrality point, the electronic dispersion relation of pristine graphene monolayers is linear and has two degenerate components, with corresponding $K$ and $K^{\prime}$ valley indices ${ }^{1}$. In the presence of a magnetic field, the effective electronic Hamiltonian for the $K$-valley reads

$$
H^{K}=v_{F} \boldsymbol{\sigma} \cdot[\boldsymbol{p}+e \boldsymbol{A}(\boldsymbol{r})]=H_{0}^{K}+V(\boldsymbol{r}),
$$

where the vector potential $\boldsymbol{A}(\boldsymbol{r})$ has been included in $H^{K}$ by minimal coupling. Here $v_{F} \approx 10^{6} \mathrm{~m} / \mathrm{s}, \boldsymbol{\sigma}$ are the Pauli matrices acting on the sublattice space, and $\boldsymbol{p}$ is the electron momentum operator. The Hamiltonian for the $K^{\prime}$ valley has a similar structure ${ }^{1}$.

In this description, a generic long-ranged disorder potential $V(\boldsymbol{r})$ is represented in both $K$ and $K^{\prime}$ valleys by

$$
V(\boldsymbol{r})=\sum_{i} \sigma_{i} V^{(i)}(\boldsymbol{r})
$$

where $i=0$ stands for scalar disorder (with $\sigma_{0}=I_{2}$ ) and $i=1,2$ for vector potential disorder, while $i=3$ represents a mass term. The focus of our study are (intrinsic and extrinsic) disordered gauge fields, associated with $V^{(1)}$ and $V^{(2)}$. In this paper we do not consider scalar disorder.

Let us introduce

$$
\begin{gathered}
\left\langle\boldsymbol{k}^{\prime} s^{\prime}|V| \boldsymbol{k} s\right\rangle=\frac{1}{2}\left[1+s s^{\prime} e^{i\left(\theta-\theta^{\prime}\right)}\right] V_{\boldsymbol{k}-\boldsymbol{k}^{\prime}}^{(0)}+ \\
\frac{s e^{i \theta}}{2}\left(V_{\boldsymbol{k}-\boldsymbol{k}^{\prime}}^{(1)}-i V_{\boldsymbol{k}-\boldsymbol{k}^{\prime}}^{(2)}\right)+\frac{s^{\prime} e^{-i \theta^{\prime}}}{2}\left(V_{\boldsymbol{k}-\boldsymbol{k}^{\prime}}^{(1)}+i V_{\boldsymbol{k}-\boldsymbol{k}^{\prime}}^{(2)}\right),
\end{gathered}
$$

where the spinor

$$
|\boldsymbol{k} s\rangle=\frac{1}{\sqrt{2 \mathcal{A}}}\left(\begin{array}{c}
1 \\
s e^{i \theta}
\end{array}\right) e^{i \boldsymbol{k} \cdot \boldsymbol{r}},
$$

is an eigenstate of $H_{0}^{K}, \theta=\tan ^{-1}\left(k_{y} / k_{x}\right), s$ indicates particle $(s=+1)$ or hole $(s=-1)$ doping, and

$$
V_{\boldsymbol{k}-\boldsymbol{k}^{\prime}}^{(i)}=\frac{1}{\mathcal{A}} \int d \boldsymbol{r} e^{i\left(\boldsymbol{k}-\boldsymbol{k}^{\prime}\right) \cdot \boldsymbol{r}} V^{(i)}(\boldsymbol{r})
$$

is the momentum representation of $V^{(i)}$. Since we deal with elastic processes, we assume in the remaining of the paper that $s=s^{\prime}$.

At low temperatures, scalar disorder (short and long ranged) is the main source of momentum relaxation in graphene systems ${ }^{2,4}$. In this paper we use a phenomenological transport time $\tau_{s}$ to account for effects of scalar disorder in the conductivity. We assume that $\tau_{s}$ is much shorter than the characteristic transport times due to random gauge fields. In Sec. IV, where we compare our results to experiments, we show that $\tau_{s}$ indeed dominates the conductivity in graphene, but some transport properties are only explained by including effects due to random gauge fields.

The ripple disorder model employed in this study is defined as follows: We describe the graphene sheet surface by $z=h(\boldsymbol{r})$, where $h$ is the surface displacement with respect to the reference plane $z=0$ at the position $\boldsymbol{r}=(x, y)$. The average of $h$ is set to zero. In line with the experiments on graphene deposited over a substrate ${ }^{9,13-16}$, we further assume that the typical heights $h_{\text {rms }}$ are much smaller that the ripple lengths $\lambda$.

We model the ripple fluctuations in $h(\boldsymbol{r})$ by the correlation function

$$
\left\langle h(\boldsymbol{r}) h\left(\boldsymbol{r}^{\prime}\right)\right\rangle=h_{\mathrm{rms}}^{2} F\left(\frac{\left|\boldsymbol{r}-\boldsymbol{r}^{\prime}\right|}{\lambda}\right),
$$

where $\langle\cdots\rangle$ denotes an average over disorder. Although theory predicts a power law height-height correlation function for free-standing membranes ${ }^{30}$, experiments support single-parameter correlations for the (static) ripples of graphene deposited over a substrate. For latter convenience, let us define

$$
h(\boldsymbol{q})=\frac{1}{\mathcal{A}} \int d \boldsymbol{r} e^{i \boldsymbol{q} \cdot \boldsymbol{r}} h(\boldsymbol{r}),
$$


where $\mathcal{A}$ is the sample size. In reciprocal space

$$
\left\langle h(\boldsymbol{q}) h\left(\boldsymbol{q}^{\prime}\right)\right\rangle=h_{\mathrm{rms}}^{2} \bar{F}(\boldsymbol{q}) \delta_{\boldsymbol{q},-\boldsymbol{q}^{\prime}},
$$

where $\bar{F}(\boldsymbol{q})$ is the Fourier transform of the correlation function $F\left(\left|\boldsymbol{r}-\boldsymbol{r}^{\prime}\right|\right)$.

We address two mechanisms that generate random magnetic fields. First, we study the case of an external strong magnetic field $\boldsymbol{B}_{\|}$applied parallel to the graphene sheet. We show that, due to the ripples, $\boldsymbol{B}_{\|}$gives rise to a random effective magnetic field $B_{\text {ext }}(\boldsymbol{r})$ perpendicular to the graphene surface. Next, we discuss the intrinsic pseudo-magnetic field $\boldsymbol{B}_{\text {int }}$ originated by the strain field corresponding to the graphene sheet profile height $h(\boldsymbol{r})$.

\section{A. Random magnetic field due to ripples and an in-plane external B-field}

Let us first consider the setup of a magnetic field applied parallel to the sample $z=0$, that has been experimentally investigated in a variety of systems ${ }^{9,23-25}$. For notational convenience, in what follows we fix the direction of $B_{\|}$along the $x$-axis, namely, $\boldsymbol{B}_{\|}=B_{\|} \hat{\boldsymbol{x}}$.

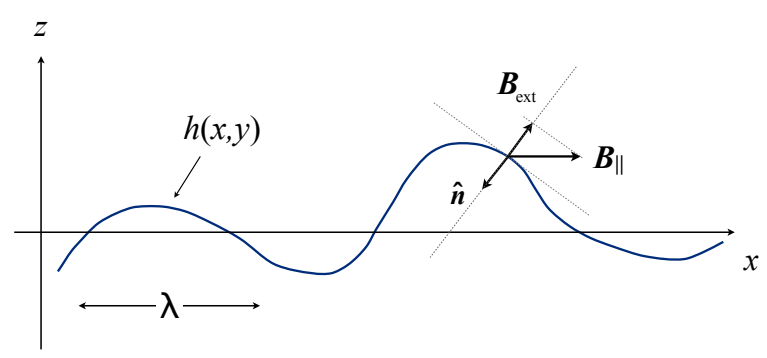

FIG. 1. Sketch of $h(\boldsymbol{r})$ along the $x$ direction. The ripple amplitudes $\delta h$ are enhanced and made comparable with $\lambda$ to help the illustration. For convenience, we take $\boldsymbol{B}_{\|}=B_{\|} \hat{\boldsymbol{x}}$.

As illustrated in Fig. 1, the parallel magnetic field $\boldsymbol{B}_{\|}$ has a component perpendicular to the surface $z=h(\boldsymbol{r})$ that is given by

$$
B_{\text {ext }}(\boldsymbol{r})=-\boldsymbol{B}_{\|} \cdot \hat{\boldsymbol{n}}(\boldsymbol{r}) .
$$

At the point $\boldsymbol{r}_{0}=\left(x_{0}, y_{0}\right)$, the surface $z=h(\boldsymbol{r})$ has a unit normal vector

$$
\hat{\boldsymbol{n}}\left(\boldsymbol{r}_{0}\right)=\left.\frac{1}{\sqrt{1+\left(\frac{\partial h}{\partial x}\right)^{2}+\left(\frac{\partial h}{\partial y}\right)^{2}}}\left(\begin{array}{c}
\partial h / \partial x \\
\partial h / \partial y \\
-1
\end{array}\right)\right|_{\boldsymbol{r}=\boldsymbol{r}_{0}} .
$$

We assume that the typical displacement magnitude is characterized by $\delta h$. For $\delta h \ll \lambda$, we write

$$
\left.\hat{\boldsymbol{n}}\left(\boldsymbol{r}_{0}\right) \approx(\partial h / \partial x, \partial h / \partial y,-1)\right|_{\boldsymbol{r}=\boldsymbol{r}_{0}} .
$$

Hence, the effective local perpendicular magnetic field reads

$$
B_{\mathrm{ext}}(\boldsymbol{r})=-\boldsymbol{B}_{\|} \cdot \nabla h(\boldsymbol{r})
$$

and is expressed, in a convenient gauge for $\boldsymbol{B}_{\|}=B_{\|} \hat{\boldsymbol{x}}$, by the vector potential

$$
A_{x}(\boldsymbol{r})=0 \quad \text { and } \quad A_{y}(\boldsymbol{r})=-B_{\|} h(\boldsymbol{r}) .
$$

Figure 2(a) illustrates a typical disorder realization of $h(\boldsymbol{r})$ with fluctuations characterized by the Gaussian correlation function $F(x)=\exp \left(-x^{2} / 2 \lambda^{2}\right)$. The corresponding magnetic field $B_{\text {ext }}(\boldsymbol{r})$, normal to the graphene sheet, is shown in Fig. 2(b). While $h(\boldsymbol{r})$ displays an isotropic disorder, $B_{\text {ext }}(\boldsymbol{r})$ is clearly anisotropic. The anisotropy direction of $B_{\text {ext }}(\boldsymbol{r})$ depends on the orientation of $B_{\|}$.

The anisotropy is quantified by inspecting the autocorrelation function

$$
\left\langle B_{\mathrm{ext}}(\boldsymbol{r}) B_{\mathrm{ext}}\left(\boldsymbol{r}^{\prime}\right)\right\rangle=B_{\|}^{2}\left\langle\frac{\partial h(\boldsymbol{r})}{\partial x} \frac{\partial h\left(\boldsymbol{r}^{\prime}\right)}{\partial x^{\prime}}\right\rangle,
$$

that can be expressed in terms of $F$ by direct differentiation. Alternatively, going to reciprocal space, one writes

$$
\begin{aligned}
\left\langle\frac{\partial h(\boldsymbol{r})}{\partial x} \frac{\partial h\left(\boldsymbol{r}^{\prime}\right)}{\partial x^{\prime}}\right\rangle & =h_{\mathrm{rms}}^{2} \sum_{\boldsymbol{q}} q_{x}^{2} \bar{F}(\boldsymbol{q}) e^{-i \boldsymbol{q} \cdot\left(\boldsymbol{r}-\boldsymbol{r}^{\prime}\right)} \\
& =-h_{\mathrm{rms}}^{2} \frac{d^{2}}{d x^{2}} F(\boldsymbol{\rho})
\end{aligned}
$$

with $\rho=\boldsymbol{r}-\boldsymbol{r}^{\prime}$.

Let us calculate $\left\langle B_{\text {ext }}(\boldsymbol{r}) B_{\text {ext }}\left(\boldsymbol{r}^{\prime}\right)\right\rangle$ for the case where $h(\boldsymbol{r})$ is characterized by a Gaussian correlation function, the same as in Fig. 2(a). The corresponding $B_{\text {ext }}(\boldsymbol{r})$ autocorrelation function reads

$$
\left\langle B_{\mathrm{ext}}(\boldsymbol{r}) B_{\mathrm{ext}}\left(\boldsymbol{r}^{\prime}\right)\right\rangle=B_{\|}^{2} \frac{h_{\mathrm{rms}}^{2}}{\lambda^{2}}\left[1-\left(\frac{\rho}{\lambda}\right)^{2} \cos ^{2} \alpha\right] e^{-\frac{\rho^{2}}{2 \lambda^{2}}}
$$

where $\alpha$ is the angle between $\boldsymbol{B}_{\|}$(or the $x$-axis) and $\boldsymbol{\rho}$.

Figure 3(a) shows the $B_{\text {ext }}(\boldsymbol{r})$ autocorrelation function obtained by averaging over $10^{5}$ ripple disorder realizations of $h(\boldsymbol{r})$, as defined by Eq. (6) with a Gaussian correlation function $F$ (for more details see, for instance, Ref. 6). As expected, it coincides with Eq. (16) and expresses the anisotropy captured by a visual inspection of Fig. 2(b).

\section{B. Pseudomagnetic field due to strain}

The out of plane deformations of a rippled membrane described by $h(\boldsymbol{r})$ can be associated with the strain tensor $u_{i j}(\boldsymbol{r})$ given by ${ }^{19,31,32}$

$$
\begin{aligned}
& u_{x x} \approx \frac{1}{2}\left(\frac{\partial h}{\partial x}\right)^{2}, \quad u_{y y} \approx \frac{1}{2}\left(\frac{\partial h}{\partial y}\right)^{2}, \quad \text { and } \\
& u_{x y} \approx \frac{1}{2}\left(\frac{\partial h}{\partial x} \frac{\partial h}{\partial y}\right) .
\end{aligned}
$$

For simplicity we have neglected the effect of in-plane deformations. 

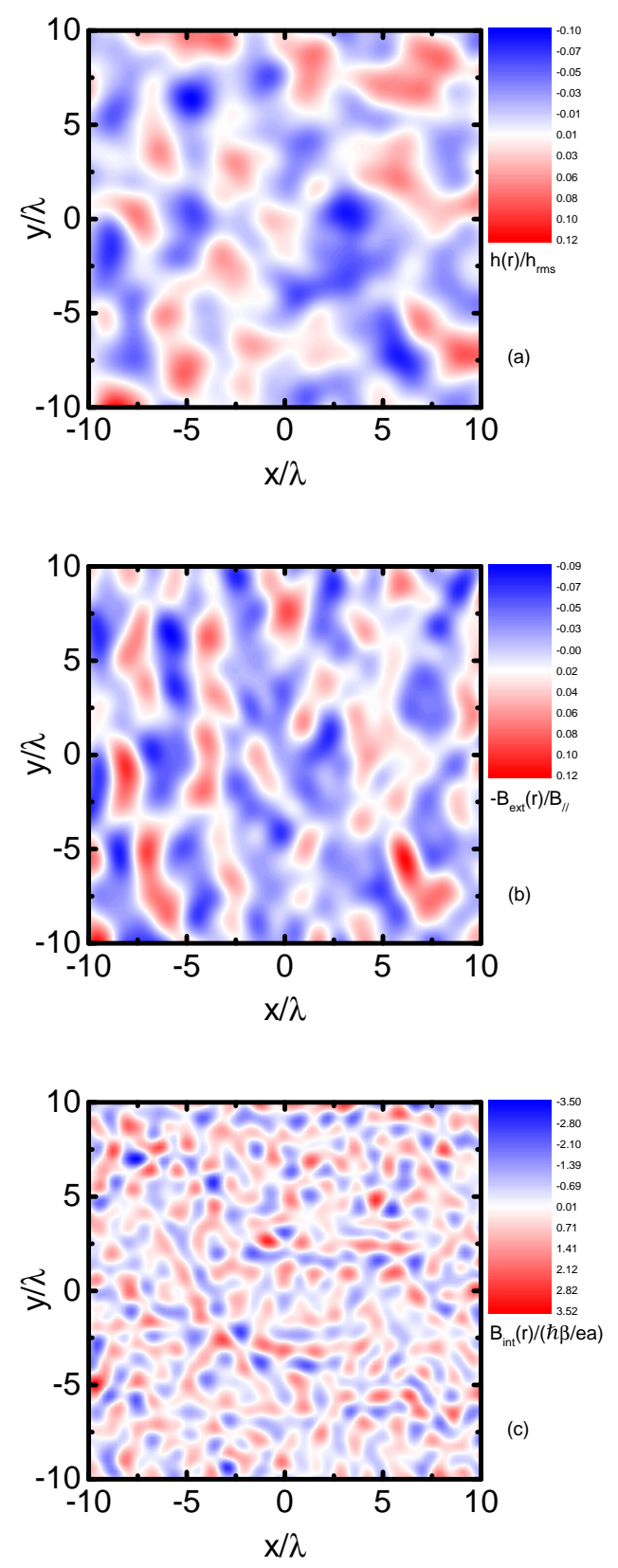

FIG. 2. (color online) Typical disorder realization of (a) $h(\boldsymbol{r})$, characterized by a Gaussian correlation function $F$, the corresponding (b) $B_{\text {ext }}(\boldsymbol{r})$ for an external $\boldsymbol{B}_{\|}$applied along the $x$ direction, defined in Eq. (12), and (c) $B_{\text {int }}(\boldsymbol{r})$ due to lattice deformations, given by Eq. (19).

The effect of strain in the low-energy electronic structure of graphene can be accounted for by introducing a scalar and a vector gauge potential in the Dirac equation $^{20,21,30}$. The scalar term reads

$$
V^{(0)}(\boldsymbol{r})=g\left[u_{x x}(\boldsymbol{r})+u_{y y}(\boldsymbol{r})\right]
$$

while, for the $K$-valley and for an armchair crystallographic orientation along the $x$-axis, $\boldsymbol{A}=\left(A_{x}, A_{y}\right)$ is
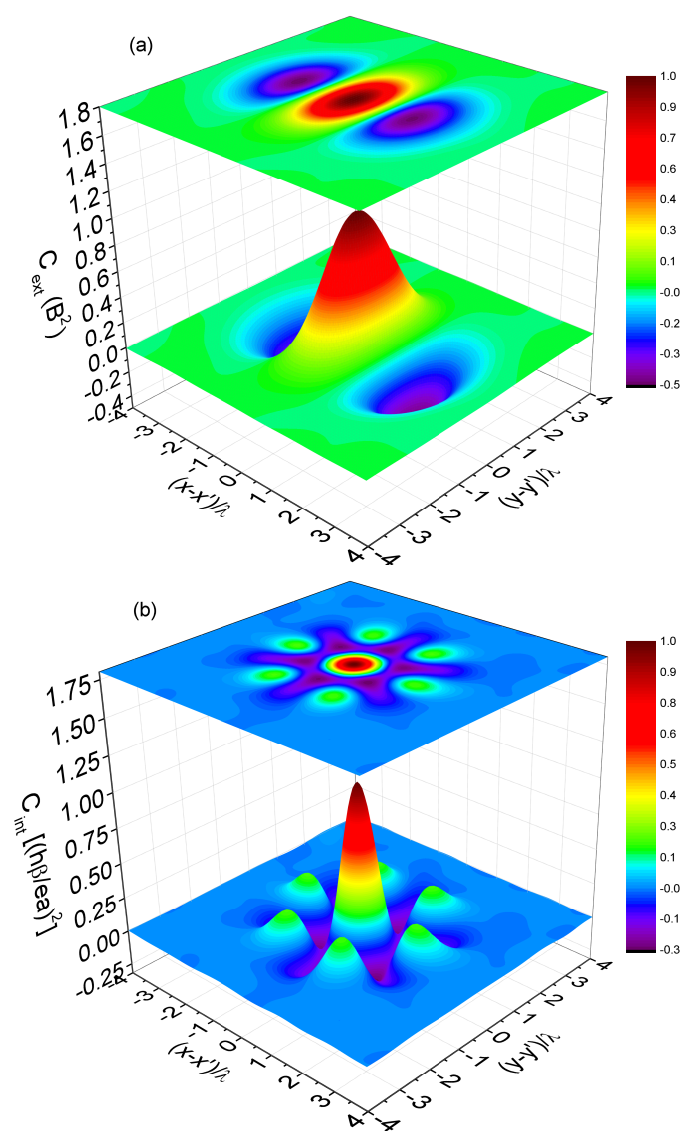

FIG. 3. (color online) Extrinsic and intrinsic magnetic field correlations functions: (a) $C_{\text {ext }}\left(\boldsymbol{r}-\boldsymbol{r}^{\prime}\right)=\left\langle B_{\text {ext }}(\boldsymbol{r}) B_{\text {ext }}\left(\boldsymbol{r}^{\prime}\right)\right\rangle$ for an external $\boldsymbol{B}_{\|}$applied along the $x$-direction and (b) $C_{\text {int }}\left(\boldsymbol{r}-\boldsymbol{r}^{\prime}\right)=\left\langle B_{\text {int }}(\boldsymbol{r}) B_{\text {int }}\left(\boldsymbol{r}^{\prime}\right)\right\rangle$ due to strain in units of $h_{\mathrm{rms}}^{4} / \lambda^{6}(\hbar \beta / e a)^{2}$, both corresponding to a ripple disordered surface $h(\mathbf{r})$ characterized by a Gaussian correlation function.

given by

$$
\begin{aligned}
& A_{x}(\boldsymbol{r})=\frac{\hbar \beta \kappa}{e a}\left[u_{x x}(\boldsymbol{r})-u_{y y}(\boldsymbol{r})\right] \\
& A_{y}(\boldsymbol{r})=-2 \frac{\hbar \beta \kappa}{e a} u_{x y}(\boldsymbol{r})
\end{aligned}
$$

where $a=1.42 \AA$ is the bond length between nearest neighboring carbon atoms, and $e$ is the electron charge. Here $g \approx 4 \mathrm{eV}, \kappa \approx 1 / 3$, and $\beta=-\partial \log t / \partial \log a \approx 2$ are dimensionless material dependent parameters ${ }^{19,31}$ that characterize the coupling between the Dirac electrons and the lattice deformations, where $t \approx 3 \mathrm{eV}$ is the hopping integral between nearest-neighbor $\pi$-orbitals.

In summary, for any given $h(\boldsymbol{r})$ one can readily calculate the pseudo-magnetic field $\boldsymbol{B}_{\text {int }}=\nabla \times \boldsymbol{A}$. Since $A_{z}=0$ and neither $A_{x}$ nor $A_{y}$ depend on $z, \boldsymbol{B}_{\text {int }}=B_{\text {int }} \hat{\boldsymbol{z}}$. Figure 2(c) shows the $B_{\text {int }}$ corresponding to the random rippled surface $h(\boldsymbol{r})$ of Fig. 2(a). Notice that the typical correlation length of $B_{\text {int }}(\boldsymbol{r})$ is much shorter than that of $h(\boldsymbol{r})$.

Let us calculate $\left\langle B_{\text {int }}(\boldsymbol{r}) B_{\text {int }}\left(\boldsymbol{r}^{\prime}\right)\right\rangle$ for a random Gaus- 
sian correlated $h(\boldsymbol{r})$, corresponding to Eq. (6) with $F(x)=e^{-x^{2} / 2 \lambda^{2}}$. To this end, we calculate the Fourier transform of the intrinsic pseudo magnetic field, namely

$$
B_{\mathrm{int}}(\boldsymbol{q})=i \frac{\hbar \beta \kappa}{e a}\left[q_{y} u_{x x}(\boldsymbol{q})+2 q_{x} u_{x y}(\boldsymbol{q})-q_{y} u_{y y}(\boldsymbol{q})\right]
$$

with

$$
u_{i j}(\boldsymbol{q})=-\frac{1}{2} \sum_{\boldsymbol{q}^{\prime}} q_{i}^{\prime}\left(q_{j}-q_{j}^{\prime}\right) h\left(\boldsymbol{q}^{\prime}\right) h\left(\boldsymbol{q}-\boldsymbol{q}^{\prime}\right)
$$

where $i$ and $j$ label the Cartesian coordinates.

We use Eqs. (20) and (21) to write the correlation function of $B_{\text {int }}$ in momentum space. The evaluation of $\left\langle B_{\text {int }}(\boldsymbol{q}) B_{\text {int }}(-\boldsymbol{q})\right\rangle$ amounts to compute the corresponding $\left\langle u_{i j}(\boldsymbol{q}) u_{i^{\prime} j^{\prime}}(-\boldsymbol{q})\right\rangle$, that result in four- $h$ correlation functions. This can be done exactly for Gaussian fluctuations and provides a good qualitative estimate for other $\operatorname{cases}^{30}$.

We obtain

$$
\left\langle B_{\text {int }}(\boldsymbol{q}) B_{\text {int }}(-\boldsymbol{q})\right\rangle=\frac{h_{\mathrm{rms}}^{4} \pi}{32 \lambda^{2} \mathcal{A}}\left(\frac{\hbar \beta \kappa}{e a}\right)^{2} q^{2}\left[16+\lambda^{4} q^{4} \sin ^{2} 3 \theta\right] e^{-\lambda^{2} q^{2} / 4},
$$

where $\theta$ is the angle between $\boldsymbol{q}$ and the $x$-direction.

By Fourier transforming back to coordinate space, we arrive at

$$
C_{\mathrm{int}}\left(\boldsymbol{r}-\boldsymbol{r}^{\prime}\right) \equiv\left\langle B_{\mathrm{int}}(\boldsymbol{r}) B_{\mathrm{int}}\left(\boldsymbol{r}^{\prime}\right)\right\rangle=\frac{h_{\mathrm{rms}}^{4}}{\lambda^{6}}\left(\frac{\hbar \beta \kappa}{e a}\right)^{2}\left[8-20 \frac{\rho^{2}}{\lambda^{2}}+9 \frac{\rho^{4}}{\lambda^{4}}-2 \frac{\rho^{6}}{\lambda^{6}} \sin ^{2} 3 \alpha\right] e^{-\rho^{2} / \lambda^{2}}
$$

where $\alpha$ is the angle between $\boldsymbol{\rho}=\boldsymbol{r}-\boldsymbol{r}^{\prime}$ and the $x$-axis. ${ }^{33}$

The correlation function $C_{\text {int }}\left(\boldsymbol{r}-\boldsymbol{r}^{\prime}\right)$ has 6 symmetry axes, reflecting the underlying graphene honeycomb lattice symmetry ${ }^{19}$. In other words, information about the graphene crystal structure survives disorder averaging. Figure 3(b) shows $C_{\text {int }}\left(\boldsymbol{r}-\boldsymbol{r}^{\prime}\right)$ obtained from $10^{5}$ numerical realizations of Gaussian correlated disorder for $h(\boldsymbol{r})$. The numerical simulations serve as a helpful test to check our analytical results. As in the previous subsection, we verify an excellent agreement within the statistical precision.

\section{DRUDE-BOLTZMANN CONDUCTIVITY}

In this Section we use the effective Dirac Hamiltonian of Eq. (1) to calculate the transport time and the DrudeBoltzmann conductivity of graphene monolayers in the presence of random gauge fields.

High mobility graphene samples have typical electronic mean free paths of $\ell \gtrsim 50 \mathrm{~nm}$. Recalling ${ }^{1}$ that the carrier density is related to the Fermi wave number by $k_{F}=\sqrt{\pi|n|}$, one readily obtains that $k_{F} \ell \gg 1$ already for a doping where $|n| \approx 10^{11} \mathrm{~cm}^{-2}$. This indicates that even for modest carrier densities a semiclassical transport description is justified. For $|n| \gtrsim 10^{11}$ $\mathrm{cm}^{-2}$ the typical graphene conductivity in good samples is much larger than $e^{2} / h$, the order of magnitude of quantum contributions to the electronic transport, such as weak localization ${ }^{2,26,27}$ and universal conduc- tance fluctuations ${ }^{34}$. In such situations, the Boltzmann approach is very successful in assessing the conductivity, as shown by direct comparison with numerical simulations using an atomistic basis ${ }^{6,35}$. As one approaches the charge neutrality point, and $k_{F} \ell \lesssim 1$, the semiclassical method is no longer suited and one has to resort to more sophisticated approaches ${ }^{2,8}$.

We now discuss how to add gauge field disorder in the Boltzmann approach. For long ranged disorder, some authors $^{36-38}$ argue that it can be advantageous to include the disorder potential in the classical Liouvillian evolution, that is, to treat $V(\boldsymbol{r})$ in the left-hand side of the Boltzmann equation. This approach is justified in the "classical" regime, where $k_{F} \lambda \gg 1$, that is, where the random fields with a characteristic length $\lambda$ vary slowly in the scale of $k_{F}^{-1}$. In graphene samples with ripple sizes $\lambda$ of the order of few to ten nanometers ${ }^{13-16}$, the latter inequality holds for a carrier density $|n| \gg 10^{12}$ $\mathrm{cm}^{-2}$, that is much larger than the doping studied in most experiments ${ }^{1}$.

In this study, we calculate transport times for both short and long ranged disorder by evaluating the corresponding Boltzmann collision integral (at the right-hand side of the equation). As mentioned in the introduction, for graphene on standard substrates (the case of interest here), the random gauge field contribution to the conductivity is not the dominant one. Hence, the electron mean free path due to ripples is larger than $\ell$ and the arguments justifying the semiclassical approximation hold.

The Boltzmann equation for graphene under a uniform 
electric field $\boldsymbol{E}$ reads $^{1,39}$

$$
-e \boldsymbol{E} \cdot \frac{\partial \varepsilon_{\boldsymbol{k}, s}}{\partial \boldsymbol{p}} \frac{\partial f_{0}}{\partial \varepsilon}=\sum_{\boldsymbol{k}^{\prime}, s^{\prime}}\left(g_{\boldsymbol{k}, s}-g_{\boldsymbol{k}^{\prime}, s^{\prime}}\right) \mathcal{W}_{\boldsymbol{k}^{\prime}, s \leftarrow \boldsymbol{k}, s}
$$

where $\varepsilon_{\boldsymbol{k}, s}=s v_{F} \hbar|\boldsymbol{k}|, f_{0}$ is the Fermi distribution function, $g_{\boldsymbol{k}}=f_{\boldsymbol{k}}-f_{0}$ is deviation from equilibrium due to the electric field, and $W_{\boldsymbol{k}^{\prime}, s^{\prime} \leftarrow \boldsymbol{k}, s}$ is the transition rate from state $(\boldsymbol{k}, s)$ to $\left(\boldsymbol{k}^{\prime}, s\right)$, that we calculate using Fermi golden rule, namely

$$
\mathcal{W}_{\boldsymbol{k}^{\prime}, s^{\prime} \leftarrow \boldsymbol{k}, s}=\frac{2 \pi}{\hbar}\left\langle\left|\left\langle\boldsymbol{k}^{\prime} s^{\prime}|V| \boldsymbol{k} s\right\rangle\right|^{2}\right\rangle \delta\left(\varepsilon_{\boldsymbol{k}, s}-\varepsilon_{\boldsymbol{k}^{\prime}, s^{\prime}}\right),
$$

where $V$ is a generic long-ranged disorder potential parametrized by Eq. (2). The $\delta$-function reflects the fact we are dealing with elastic processes and, hence, $s=s^{\prime}$. In our model, the transition rates do not depend on $s$. Accordingly we drop this index whenever its omission does not introduce an ambiguity.

The scattering processes we address are anisotropic. The calculation of the transport properties in this case is slightly different ${ }^{40,41}$ than that of the standard isotropic case $^{39}$. In this study, we adapt the nice method developed by Tokura ${ }^{42}$ - that is briefly described in what follows - to calculate the transport times of massless Dirac electrons in graphene.

In both situation of interest, the scattering potential correlation functions have at least one symmetry axis. For convenience, we choose the $x$-axis along a symmetry axis and define ${ }^{42}$

$$
g_{\boldsymbol{k}}=\left(-\frac{\partial f_{0}}{\partial \varepsilon_{\boldsymbol{k}}}\right) e v_{\boldsymbol{k}} \boldsymbol{\tau}(\theta) \cdot \boldsymbol{E},
$$

where $\boldsymbol{\tau}(\theta)$ is the relaxation time vector to be solved. We recall that $\theta$ is the angle between $\boldsymbol{k}$ and the $x$-axis. Note that $\boldsymbol{\tau}$ depends explicitly on $\theta$ and implicitly on $|\boldsymbol{k}|$.

The current density (spin and valley degeneracies included) is given by

$$
\begin{aligned}
\boldsymbol{j} & =\frac{4}{\mathcal{A}} \sum_{\boldsymbol{k}} e \boldsymbol{v}_{\boldsymbol{k}} g_{\boldsymbol{k}} \\
& =\frac{e^{2}}{\pi^{2}} \int_{0}^{\infty} d k k\left(-\frac{\partial f_{0}}{\partial \varepsilon_{\boldsymbol{k}}}\right) \int_{0}^{2 \pi} d \theta v_{\boldsymbol{k}} \boldsymbol{v}_{\boldsymbol{k}}[\boldsymbol{\tau}(\theta) \cdot \boldsymbol{E}],
\end{aligned}
$$

from which one obtains the conductivity tensor

$$
\sigma=\frac{e^{2}\left|\varepsilon_{F}\right|}{\hbar^{2} \pi^{2}} \int_{0}^{2 \pi} d \theta\left(\begin{array}{cc}
\tau_{x}(\theta) \cos \theta & \tau_{y}(\theta) \cos \theta \\
\tau_{x}(\theta) \sin \theta & \tau_{y}(\theta) \sin \theta
\end{array}\right),
$$

where $\varepsilon_{F}$ is the Fermi energy, measured with respect to the charge neutrality point energy. For the sake of simplicity, in Eq. (28) we have taken the zero-temperature limit, namely, $-\partial f_{0} / \partial \varepsilon=\delta\left(\varepsilon-\varepsilon_{F}\right)$.

By substituting the ansatz (26) in the Boltzmann equation, Eq. (24), one obtains an integral equation for $\boldsymbol{\tau}(\theta)$, namely

$$
\begin{aligned}
& \cos \theta=\int_{0}^{2 \pi} d \theta^{\prime}\left[\tau_{x}(\theta)-\tau_{x}\left(\theta^{\prime}\right)\right] \mathcal{W}\left(\theta, \theta^{\prime}\right) \\
& \sin \theta=\int_{0}^{2 \pi} d \theta^{\prime}\left[\tau_{y}(\theta)-\tau_{y}\left(\theta^{\prime}\right)\right] \mathcal{W}\left(\theta, \theta^{\prime}\right),
\end{aligned}
$$

where $\theta^{\prime}$ is the angle between $\boldsymbol{k}^{\prime}$ and the $x$-axis, and

$$
\begin{aligned}
\mathcal{W}\left(\theta, \theta^{\prime}\right) & =\frac{\mathcal{A}}{(2 \pi)^{2}} \int_{0}^{\infty} d k^{\prime} k^{\prime} \mathcal{W}_{\boldsymbol{k}^{\prime}, s \leftarrow \boldsymbol{k}, s} \\
& =\frac{\mathcal{A}\left|\varepsilon_{F}\right|}{2 \pi v_{F}^{2} \hbar^{3}}\left\langle\left|\left\langle\boldsymbol{k}^{\prime}, s|V| \boldsymbol{k}, s\right\rangle\right|^{2}\right\rangle,
\end{aligned}
$$

where, due to the zero-temperature limit, $k=k^{\prime}=k_{F}$.

The matrix element $\left\langle\boldsymbol{k}^{\prime}, s|V| \boldsymbol{k}, s\right\rangle$ depends on $\boldsymbol{q}=\boldsymbol{k}-$ $\boldsymbol{k}^{\prime}$ and $\varphi=\pi / 2+\left(\theta+\theta^{\prime}\right) / 2$, the angle between $\boldsymbol{q}$ and the $x$-axis. Hence, $\mathcal{W}\left(\theta, \theta^{\prime}\right)$ is better cast as $\mathcal{W}\left(q_{\zeta}, \varphi\right)$. We use the standard notation $\zeta=\left|\theta-\theta^{\prime}\right|$ and $q_{\zeta}=$ $2 k_{F} \sin (\zeta / 2)$.

By expressing $\tau_{x}$ and $\tau_{y}$ in terms of a Fourier series, one transforms Eqs. (29) and (30) into an (infinite) set of algebraic equations. Using the $x$-axis symmetry and that $\mathcal{W}\left(\theta, \theta^{\prime}\right)=\mathcal{W}\left(\theta^{\prime}, \theta\right)^{43}$, one shows that ${ }^{42}$

$$
\begin{aligned}
\tau_{x}(\theta) & =\sum_{n=1}^{\infty} \tau_{x}^{(n)} \cos [(2 n-1) \theta] \\
\tau_{y}(\theta) & =\sum_{n=1}^{\infty} \tau_{y}^{(n)} \sin [(2 n-1) \theta] .
\end{aligned}
$$

By inserting the above relations in Eq. (28), one concludes that the conductivity tensor is diagonal, with

$$
\sigma_{x x}=\frac{e^{2}\left|\varepsilon_{F}\right|}{\hbar^{2} \pi} \tau_{x}^{(1)} \quad \text { and } \quad \sigma_{y y}=\frac{e^{2}\left|\varepsilon_{F}\right|}{\hbar^{2} \pi} \tau_{y}^{(1)},
$$

that supports the interpretation of $\boldsymbol{\tau}^{(1)}$ as a transport time vector.

The symmetry $\mathcal{W}\left(\theta, \theta^{\prime}\right)=\mathcal{W}\left(\theta^{\prime}, \theta\right)$ implies that $\mathcal{W}\left(q_{\zeta}, \varphi\right)=\mathcal{W}\left(q_{\zeta}, \varphi+\pi\right)=\mathcal{W}\left(q_{\zeta}, \varphi-\pi\right)$. In turn

$$
\mathcal{W}\left(q_{\zeta}, \varphi\right)=\sum_{n=0}^{\infty} \mathcal{W}_{n}\left(q_{\zeta}\right) \cos (2 n \varphi)
$$

with an obvious inversion relation.

By using the Fourier expansions for $\boldsymbol{\tau}(\theta)$ and $W\left(q_{\zeta}, \varphi\right)$, Eqs. (29) and (30) can be cast in matrix form ${ }^{42}$

$$
\delta_{l, 1}=\sum_{n=1}^{\infty} M_{l, n}^{-} \tau_{x}^{(n)} \quad \text { and } \quad \delta_{l, 1}=\sum_{n=1}^{\infty} M_{l, n}^{+} \tau_{y}^{(n)},
$$

where the matrix elements of $M^{ \pm}$are $e^{42}$

$$
M_{l, n}^{ \pm}=\frac{(-1)^{l-n}}{2}\left[\left(1+\delta_{l, n}\right) J_{|l-n|, n+l-1} \pm J_{n+l-1,|l-n|}\right],
$$

with

$$
J_{n, m}=\int_{0}^{2 \pi} d \zeta \mathcal{W}_{n}\left(q_{\zeta}\right)[\cos (n \zeta)-\cos (m \zeta)] .
$$

Finally, by inverting $M^{ \pm}$in Eq. (36), one writes the vector transport time components as

$$
\tau_{x}^{(1)}=\left[\left(M^{-}\right)^{-1}\right]_{11} \quad \text { and } \quad \tau_{y}^{(1)}=\left[\left(M^{+}\right)^{-1}\right]_{11} .
$$


Note that for isotropic scattering, all $\mathcal{W}_{n}$ with $n>$ 0 are zero and $M^{ \pm}$is diagonal, with elements $K_{l, l}=$ $J_{0,2 l-1}$. Hence, the vector transport time components coincide, $\tau_{x}^{(1)}=\tau_{y}^{(1)}=\tau^{(1)}$, and read

$$
\frac{1}{\tau^{(1)}}=J_{0,1}=\int_{0}^{2 \pi} d \zeta \mathcal{W}_{0}\left(q_{\zeta}\right)(1-\cos \zeta),
$$

which is the standard expression for the transport time in isotropic systems.

\section{A. Effect of an in-plane magnetic field}

Let us now calculate the effect of an external parallel magnetic field on the conductivity. From Eq. (13) we write the effective disorder potential for the $K$-valley as

$$
V_{\mathrm{ext}}(\boldsymbol{r})=v_{F} e \sigma_{y} A_{y}(\boldsymbol{r})=-v_{F} e B_{\|} h(\boldsymbol{r}) \sigma_{y} .
$$

We recall that $h(\boldsymbol{r})$ varies slowly in the scale of the lattice spacing and, hence, $V_{\text {ext }}(\boldsymbol{r})$ is long-ranged and does not mix valleys.

The momentum relaxation rate $W_{\boldsymbol{k}^{\prime} \leftarrow \boldsymbol{k}}$ reads

$$
\mathcal{W}_{\boldsymbol{k}^{\prime} \leftarrow \boldsymbol{k}}=\delta\left(k-k^{\prime}\right) \frac{2 \pi e^{2} v_{F}}{\hbar^{2}} B_{\|}^{2} \sin ^{2}\left(\frac{\theta+\theta^{\prime}}{2}\right) \frac{C_{h}(q)}{\mathcal{A}} .
$$

where

$$
C_{h}(q)=\int d \boldsymbol{r} e^{i \boldsymbol{q} \cdot \boldsymbol{r}}\langle h(0) h(\boldsymbol{r})\rangle
$$

is the form factor of the height-height correlation function. Here $\langle\cdots\rangle$ indicates disorder average.

From Eq. (31) we obtain

$$
\mathcal{W}(q, \varphi)=\frac{\left(e B_{\|}\right)^{2}\left|\varepsilon_{F}\right|}{4 \pi \hbar^{3}} C_{h}(q)(1+\cos 2 \varphi),
$$

that has only 2 non-zero Fourier components, namely,

$$
\mathcal{W}_{n}(q)=\frac{\left(e B_{\|}\right)^{2}\left|\varepsilon_{F}\right|}{4 \pi \hbar^{3}} C_{h}(q), \quad \text { for } \quad n=0,1
$$

while $\mathcal{W}_{n}(q)=0$ for $n \geq 2$.

In this case, the $M^{ \pm}$matrix is tridiagonal and reads ${ }^{42}$

$$
M^{ \pm}=\left(\begin{array}{cccc}
\left(1 \mp \frac{1}{2}\right) J_{0,1} & -\frac{1}{2} J_{1,2} & 0 & \cdots \\
-\frac{1}{2} J_{1,2} & J_{0,3} & -\frac{1}{2} J_{1,4} & \cdots \\
0 & -\frac{1}{2} J_{1,4} & J_{0,5} & \cdots \\
\vdots & \vdots & \vdots & \ddots
\end{array}\right) .
$$

The inverse transport time components are given by

$$
\frac{1}{\tau_{x}^{(1)}}=\frac{3}{2} J_{0,1}-\Gamma_{3} \quad \text { and } \quad \frac{1}{\tau_{y}^{(1)}}=\frac{1}{2} J_{0,1}-\Gamma_{3},
$$

where $\Gamma_{3}$ is isotropic and determined by the continued fraction relation

$$
\Gamma_{m}=\frac{\left(J_{1, m-1}\right)^{2}}{4\left(J_{0, m}-\Gamma_{m+2}\right)} .
$$

In practice, we compute $\Gamma_{3}$ by assuming that $\Gamma_{\bar{m}}=0$ and subsequent iteration of (48). The choice of $\bar{m}$ determines the precision of the calculation: The larger $\bar{m}$, the more accurate is $\Gamma_{3}$.

Assuming that $\Gamma_{3} \ll J_{0,1}$ leads to an interesting result, that is

$$
\frac{1}{\tau_{y}^{(1)}}=\frac{1}{3 \tau_{x}^{(1)}}=\frac{\left(e B_{\|}\right)^{2}\left|\varepsilon_{F}\right|}{8 \pi \hbar^{3}} \int_{0}^{2 \pi} d \zeta(1-\cos \zeta) C_{h}(q)
$$

In this limit $\tau_{y}^{(1)} / \tau_{x}^{(1)}=3$. In other words, for $\Gamma_{3} \ll J_{0,1}$ the corrections to the conductivity due to $\boldsymbol{B}_{\|}$lead to $\Delta \sigma_{y y}=3 \Delta \sigma_{x x}$, regardless of the dependence of correlation function $C_{h}(q)$ on $q$.

For $\Gamma_{3}=0$ and $C_{h}(q)=2 \pi \lambda^{2} h_{\mathrm{rms}}^{2} e^{-\lambda^{2} q^{2} / 2}$, we write $\tau_{x, y}^{(1)}$ in closed analytical form, namely

$$
\begin{aligned}
\frac{1}{\tau_{y}^{(1)}}=\frac{1}{3 \tau_{x}^{(1)}}= & \frac{\left(e B_{\|}\right)^{2}\left|\varepsilon_{F}\right|}{4 \hbar^{3}}\left(\lambda h_{\mathrm{rms}}\right)^{2} \\
& \times e^{-\lambda^{2} k_{F}^{2}}\left[I_{0}\left(\lambda^{2} k_{F}^{2}\right)-I_{1}\left(\lambda^{2} k_{F}^{2}\right)\right],
\end{aligned}
$$

where $I_{0}$ and $I_{1}$ are modified Bessel functions of the first kind. We use $k_{F}=\sqrt{\pi|n|}$ to express the conductivity in terms of the charge carrier density $n$. We conclude that the correction to the conductivity due to an in-plane magnetic field depends quadratically on $h_{\text {rms }} B_{\|}$and has a non-trivial dependence on $\lambda^{2}|n|$.

In the high doping limit of $\lambda|n|^{1 / 2} \gg 1$, Eq. (50) gives the resistivity contribution of an in-plane magnetic field in a rippled graphene sheet

$$
\Delta \rho_{y y}=3 \Delta \rho_{x x} \approx \frac{1}{2 \sqrt{2} \hbar} \frac{h_{\mathrm{rms}}^{2}}{\lambda} B_{\|}^{2}|n|^{-3 / 2}
$$

in agreement with Ref. 9.

Figure 4 shows the resistivity $\Delta \rho_{y y}$ versus the carrier density $n$ (due to particle hole symmetry, we only show $n>0$ ) for $\bar{m}=3$ and $\bar{m} \rightarrow \infty$. The optimal $\bar{m}$ value to obtain convergence depends on $\lambda^{2} n$. The inset shows $\Delta \rho_{y y}$ for $\lambda^{2} n$ values outside the validity range of the asymptotic expansion. As discussed in the next section, the $\lambda^{2} n$ range displayed in the inset corresponds to the typical experimental situation. We find that the $|n|^{-3 / 2}$ scaling predicted by the asymptotic expansion (51) is only a rough approximation.

In general, $\Gamma_{3}$ is a non-vanishing correction to the transport time components, hence $\tau_{y}^{(1)} / \tau_{x}^{(1)} \neq 3$. However, for Gaussian ripple height correlations, the ratio $\tau_{y}^{(1)} / \tau_{x}^{(1)}$ is a function only of $\lambda k_{F}$.

Figure 5 shows $\tau_{y}^{(1)} / \tau_{x}^{(1)}$ versus $\lambda^{2} n$. It illustrates the importance of $\Gamma_{3}$ in the calculation of the conductivity corrections. We find that by increasing the carrier concentration the anisotropic conductivity is considerably favored.

The transport properties predicted by Eq. (50) are only slightly modified for the case of exponential ripple heigh correlations, $\langle h(0) h(\boldsymbol{r})\rangle=h_{\mathrm{rms}}^{2} e^{-r / \lambda}$ : For $\lambda|n|^{1 / 2} \gg 1$, the resistivity tensor given by Eq. (51) is multiplied ${ }^{44}$ by a prefactor of order of unity times $\log \left(\lambda^{2}|n|\right)$. 


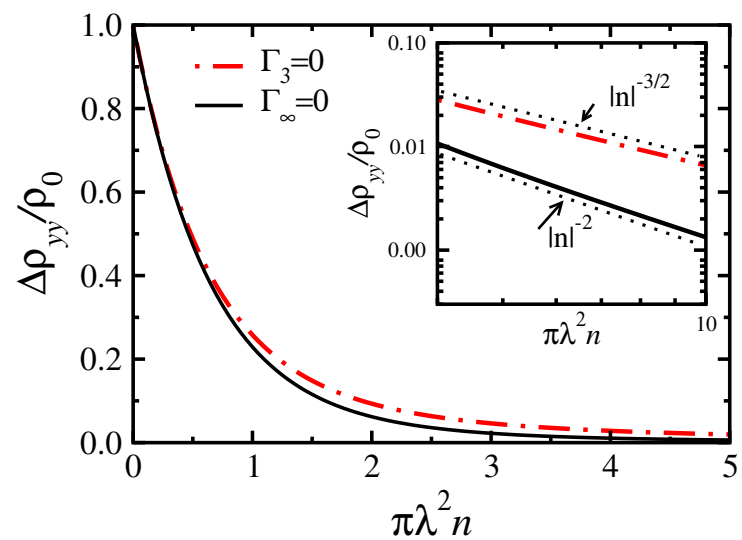

FIG. 4. (color online) Resistivity $\Delta \rho_{y y}$ in units of $\rho_{0}=$ $\left(\pi \lambda h_{\mathrm{rms}} B_{\|}\right)^{2} / 2 \hbar$ due to $\boldsymbol{B}_{\|}$as a function of $\lambda^{2} n$. Inset: The same as in the main plot in log-log scale to illustrate the dependence of $\Delta \rho_{x x}$ on $|n|$.

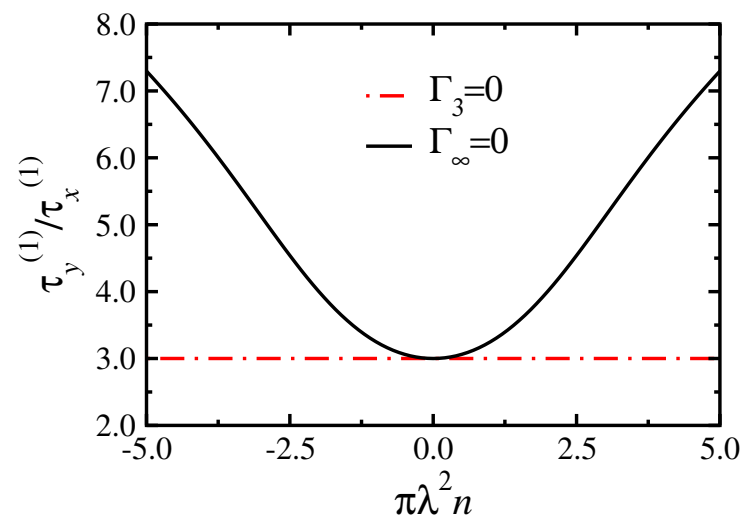

FIG. 5. (color online) Ratio $\tau_{y}^{(1)} / \tau_{x}^{(1)}$ as a function of $\pi \lambda^{2} n$. For $\pi \lambda^{2} n<5.0$, the limit $\bar{m} \rightarrow \infty$ is attained within $10^{-4}$ accuracy for $\bar{m}=13$.

\section{B. Effect of strain fields}

Let us now consider the effect of the pseudo-magnetic fields due to strain, $B_{\text {int }}$, in the conductivity of monolayer graphene sheets. In contrast to the mechanism discussed above, $B_{\text {int }}(\boldsymbol{r})$ is solely determined by $h(\boldsymbol{r})$ and the material properties. Hence, it intrinsic to any graphene sample with disordered ripples.

We use Eq. (19) to calculate the vector transport time $\tau$ for the intrinsic effective vector potential due to strain. For the $K$-valley $V_{\text {int }}$ reads

$$
V_{\mathrm{int}}(\boldsymbol{r})=\hbar v_{F} \frac{\beta \kappa}{a}\left\{\left[u_{x x}(\boldsymbol{r})-u_{y y}(\boldsymbol{r})\right] \sigma_{x}-2 u_{x y}(\boldsymbol{r}) \sigma_{y}\right\}
$$

In contrast with the previous subsection, here it is difficult to make quantitative progress without assuming a specific form for the ripple height correlation function. The qualitative behavior of the conductivity corrections due to strain that has been reported in the literature ${ }^{30}$ is not sufficient for the analysis we propose.

As in Sec. II B, we calculate $\left\langle u_{i j}(\boldsymbol{r}) u_{i^{\prime} j^{\prime}}\left(\boldsymbol{r}^{\prime}\right)\right\rangle$ by assuming Gaussian correlated ripple height fluctuations, $C_{h}(q)=2 \pi \lambda^{2} h_{\mathrm{rms}}^{2} e^{-\lambda^{2} q^{2} / 2}$. After some lengthy but straightforward algebra, we obtain

$$
\begin{aligned}
\langle|\left\langle\boldsymbol{k}^{\prime} s\right| V_{\mathrm{int}} & \left.\left.|\boldsymbol{k} s\rangle\right|^{2}\right\rangle=\frac{v_{F}^{2} \hbar^{2} \beta^{2} \kappa^{2}}{32 a^{2}} \frac{\pi h_{\mathrm{rms}}^{4}}{\lambda^{2} \mathcal{A}} \\
\times & \left\{16+\lambda^{4} q^{4} \cos ^{2}\left[\frac{3}{2}\left(\theta+\theta^{\prime}\right)\right]\right\} e^{-\lambda^{2} q^{2} / 4} .
\end{aligned}
$$

Using Eq. (31) we arrive at

$$
\mathcal{W}(q, \varphi)=\mathcal{W}_{\text {int }}\left[16+\frac{\lambda^{4} q^{4}}{2}(1+\cos 6 \varphi)\right] e^{-\lambda^{2} q^{2} / 4}
$$

where

$$
\mathcal{W}_{\mathrm{int}}=\frac{\beta^{2} \kappa^{2}\left|\varepsilon_{F}\right|}{64 a^{2} \hbar} \frac{h_{\mathrm{rms}}^{4}}{\lambda^{2}} .
$$

(The notation is the same as that of the previous section.) The only non-zero Fourier components of $\mathcal{W}(q, \varphi)$ are

$$
\begin{aligned}
& \mathcal{W}_{0}(q)=\mathcal{W}_{\text {int }}\left(16+\frac{\lambda^{4} q^{4}}{2}\right) e^{-\lambda^{2} q^{2} / 4} \text { and } \\
& \mathcal{W}_{3}(q)=\mathcal{W}_{\text {int }} \frac{\lambda^{4} q^{4}}{2} e^{-\lambda^{2} q^{2} / 4} .
\end{aligned}
$$

In this case, the $M^{ \pm}$matrix reads ${ }^{42}$

$$
M^{ \pm}=\left(\begin{array}{ccccc}
J_{0,1} & 0 & \mp \frac{1}{2} J_{3,2} & -\frac{1}{2} J_{3,4} & \cdots \\
0 & J_{0,3} \mp \frac{1}{2} J_{3,0} & 0 & 0 & \cdots \\
\mp \frac{1}{2} J_{3,2} & 0 & J_{0,5} & 0 & \cdots \\
-\frac{1}{2} J_{3,4} & 0 & 0 & J_{0,7} & \cdots \\
\vdots & \vdots & \vdots & \vdots & \ddots
\end{array}\right),
$$

and the inverse transport time components are given by

$$
\frac{1}{\tau_{x / y}^{(1)}}=J_{0,1}-\frac{\left(J_{3,2}\right)^{2}}{4 J_{0,5}}-\frac{\left(J_{3,4}\right)^{2}}{4 J_{0,7}}+\cdots
$$

Since $\tau_{x}^{(1)}=\tau_{y}^{(1)}$, the conductivity corrections due to the strain field are isotropic. This result seems to be at odds with the fact that the pseudo-magnetic field autocorrelation function $\left\langle B_{\text {int }}(\boldsymbol{r}) B_{\text {int }}\left(\boldsymbol{r}^{\prime}\right)\right\rangle$ clearly shows an hexagonal symmetry, as illustrated by Fig. 3 b. As shown by Tokura $^{42}$, using general arguments, this is a false paradox: The conductivity tensor becomes anisotropic only for scattering processes characterized by a single symmetry axis, like in the $B_{\|}$case, analyzed in the previous subsection.

Assuming that $J_{0,1}$ vastly dominates the sum in Eq. (58), we obtain 


$$
\frac{1}{\tau_{x / y}^{(1)}}=\mathcal{W}_{\mathrm{int}} \pi e^{-\lambda^{2} k_{F}^{2} / 2}\left[\left(32+8 \lambda^{2} k_{F}^{2}+16 \lambda^{4} k_{F}^{4}\right) I_{0}\left(\lambda^{2} k_{F}^{2} / 2\right)-\left(64+24 \lambda^{2} k_{F}^{2}+16 \lambda^{4} k_{F}^{4}\right) I_{1}\left(\lambda^{2} k_{F}^{2} / 2\right)\right]
$$

where $I_{0}$ and $I_{1}$ are modified Bessel functions of the first kind.

In the limit of $\lambda|n|^{-1} \gg 1$, we obtain the correction to the resistivity

$$
\Delta \rho_{x x}=\Delta \rho_{y y} \approx \frac{h}{e^{2}} \frac{23 \beta^{2} \kappa^{2}}{32 \pi} \frac{h_{\mathrm{rms}}^{4}}{\lambda^{2} a^{2}} \lambda^{-3}|n|^{-3 / 2} .
$$

The above asymptotic leading order expansion for $\Delta \rho$ helps us to develop some insight on the relevant parameters. However, since the situation of $k_{F} \lambda \gg 1$ is hardly met in the current experimental situations of interest, it is necessary to numerically calculate the inverse transport times, as we do in the next subsection. As expected the strain corrections to the conductivity depend on material parameters, and are a non-trivial function of $\lambda, h_{\mathrm{rms}}$, and $|n|$. Since these corrections are small compared to other disorder effects, they are difficult to be noticed in standard transport experiments. This situation changes if we consider the combined effect of intrinsic and extrinsic random magnetic fields, as we discuss in the next.

\section{Combined effect of extrinsic and intrinsic random magnetic fields}

We conclude this section by analyzing the combined effect of both previously discussed sources of random magnetic field disorder. As before, we assume that the sys- tem transport properties are dominated by other scattering processes, with a corresponding (isotropic) transport time $\tau_{\mathrm{s}}$.

It is customary to use Matthiessen's rule when dealing with systems characterized by different competing relaxation time mechanisms. In our case, Matthiessen's rule translates into adding the inverse transport times given by Eqs. (47) and (58), namely

$$
\frac{1}{\tau_{x / y}^{(1)}}=\frac{2 \pm 1}{2} J_{0,1}^{\text {ext }}-\Gamma_{3}^{\text {ext }}+J_{0,1}^{\text {int }}-\frac{\left(J_{3,2}^{\text {int }}\right)^{2}}{4 J_{0,5}^{\text {int }}}-\frac{\left(J_{3,4}^{\text {int }}\right)^{2}}{4 J_{0,7}^{\text {int }}}+\cdots
$$

This naive approach was shown to be inaccurate when dealing with anisotropic potentials ${ }^{42}$.

We analyze the combined effect of intrinsic and extrinsic random magnetic fields by considering an effective $M$-matrix given by

$$
M_{\mathrm{tot}}^{ \pm}=M_{\mathrm{ext}}^{ \pm}+M_{\mathrm{int}}^{ \pm}
$$

where $M_{\mathrm{ext}}^{ \pm}$and $M_{\mathrm{int}}^{ \pm}$are given by Eqs. (46) and (57) respectively.

The $\boldsymbol{\tau}$ components are

$$
\tau_{x}^{(1)}=\left[\left(M_{\text {tot }}^{-}\right)^{-1}\right]_{11} \quad \text { and } \quad \tau_{y}^{(1)}=\left[\left(M_{\text {tot }}^{+}\right)^{-1}\right]_{11},
$$

that, for $\bar{m}=5$, explicitly read

$$
\begin{aligned}
\frac{1}{\tau_{x / y}^{(1)}}= & \left(1 \pm \frac{1}{2}\right) J_{0,1}^{\text {ext }}+J_{0,1}^{\text {int }}+\frac{1}{\left(J_{0,3}^{\text {ext }}+J_{0,3}^{\text {int }} \mp J_{3,0}^{\text {int }} / 2\right)\left(J_{0,5}^{\text {ext }}+J_{0,5}^{\text {int }}\right)-\left(J_{1,4}^{\text {ext }}\right)^{2} / 4}\left[-\frac{1}{4}\left(J_{0,5}^{\text {ext }}+J_{0,5}^{\text {int }}\right)\left(J_{1,2}^{\text {ext }}\right)^{2}\right. \\
& \left.\mp \frac{1}{4} J_{3,2}^{\text {int }} J_{1,2}^{\text {ext }} J_{1,4}^{\text {ext }}-\frac{1}{4}\left(J_{3,2}^{\text {int }}\right)^{2}\left(J_{0,3}^{\text {ext }}+J_{0,3}^{\text {int }} \mp \frac{1}{2} J_{3,0}^{\text {int }}\right)\right]+\cdots
\end{aligned}
$$

This result is clearly different from Eq. (61), since it mixes intrinsic and extrinsic effects. Let us now discuss the dependence of $\boldsymbol{\tau}$ on $B_{\|}, \lambda, h_{\mathrm{rms}}$, and $n$. For that purpose we numerically invert the matrix $M_{\text {tot }}^{ \pm}$, at order $\bar{m} \approx 30-50$ to guarantees an accuracy of $10^{-5}$ for the analyzed parameter range.

The resistivity corrections obtained from the Matthiessen's rule, Eq. (61) depend quadratically on $B_{\|}$, in line with the experiment ${ }^{9}$. The full $M$-matrix analysis does not guarantee this simple dependence. In Fig. 6 we plot the resistivity correction $\Delta \rho_{y y}$ calculated using the full $M$-matrix and compare it with the one obtained from the Matthiessen rule, given by Eq. (61), for realistic values of $h_{\mathrm{rms}}, \lambda$, and $n$. The full
$M$-matrix calculation (indicated as "exact") shows an overall higher resistivity than that obtained from the Matthiessen rule. It depends linearly on $B_{\|}^{2}$ for large $B_{\|}$and deviates from this dependence only when $B_{\|}$ becomes small.

In contrast, the dependence of $\Delta \rho$ with $h_{\mathrm{rms}}, \lambda$, and $|n|$ is not trivial. For $h_{\text {rms }}$ and $\lambda$ values taken close to the ones reported by topography experiments ${ }^{13-16}$, a numerical study using the full $M$-matrix approach gives $\Delta \rho_{y y} \propto \lambda^{-\alpha}$ with $\alpha \approx 3 \cdots 4, \Delta \rho_{y y} \propto h_{\text {rms }}^{\beta}$ with $\beta \approx 3$, and $\Delta \rho_{y y} \propto|n|^{-\gamma}$ with $\gamma \approx 2$. In summary, $\Delta \rho$ is very sensitive on small variations of $h_{\mathrm{rms}}$ and $\lambda$.

In Fig. 7 we compare Eqs. (47), (61), and (64) to gain insight on how the strain mechanism affects the ratio 


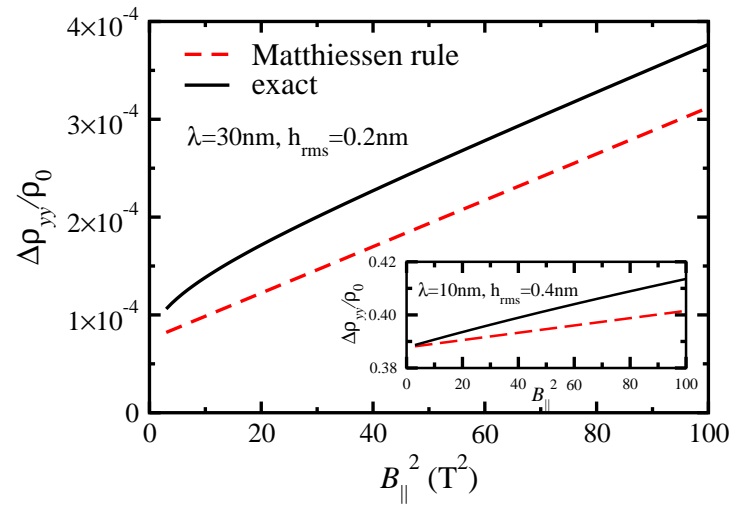

FIG. 6. (color online) Resistivity correction $\Delta \rho_{y y}$ in units of $\rho_{0}=\left(\pi \lambda h_{\mathrm{rms}} B_{\|}\right)^{2} / 2 \hbar$ as a function of the in-plane magnetic field $B_{\|}$for $h_{\mathrm{rms}}=0.2 \mathrm{~nm}, \lambda=30 \mathrm{~nm}$, and $n=10^{12} \mathrm{~cm}^{-2}$. Inset: The same as in the main figure for $h_{\mathrm{rms}}=0.4 \mathrm{~nm}$ and $\lambda=10 \mathrm{~nm}$.

$\tau_{y}^{(1)} / \tau_{x}^{(1)}$. We find that the strain fields contribute to a strong suppression of the anisotropy in the transport time due to a strong $B_{\|}$. However, for realistic parameter values the anisotropy is still very large and of the order of $\tau_{y}^{(1)} / \tau_{x}^{(1)} \approx 10$ for $|n| \approx 10^{12} \mathrm{~cm}^{-2}$.

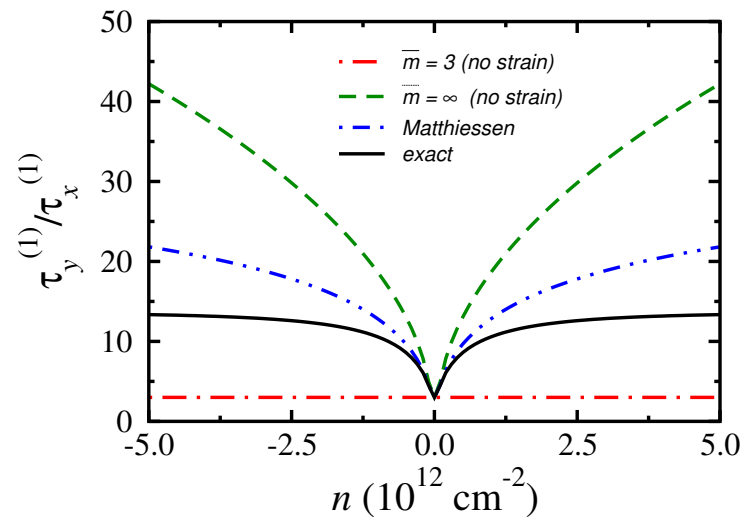

FIG. 7. (color online) Anisotropy $\tau_{y}^{(1)} / \tau_{x}^{(1)}$ as a function of the carrier concentration $n$ using different approximation schemes, for $h_{\mathrm{rms}}=0.2 \mathrm{~nm}, \lambda=30 \mathrm{~nm}$, and $B_{\|}=8 \mathrm{~T}$. The red and green lines stand for the contribution of $B_{\|}$without accounting for strain fields. The blue line represents the contributions of both external and strain fields using the conventional Matthiessen's rule. The black line stands for the combined effect of intrinsic and extrinsic fields obtained for the full $M$-matrix analysis.

In order to further compare our results with the experiment $^{9}$, let us introduce the magnetoresistance $\Delta \rho=\boldsymbol{E} \cdot \boldsymbol{j} / j^{2}$, where $j_{x}=j \cos \xi, j_{y}=j \sin \xi$, and $\xi$ is the angle between $\boldsymbol{B}_{\|}$and $\boldsymbol{j}$. Using the relation $E_{i}=\rho_{i j} J_{j}$ one writes $^{45}$

$$
\Delta \rho(\xi)=\Delta \rho_{x x} \cos ^{2} \xi+\Delta \rho_{y y} \sin ^{2} \xi .
$$

Ref. 9 reports $\Delta \rho\left(70^{\circ}\right) / \Delta \rho\left(20^{\circ}\right) \approx 0.13-0.26$. Using $\lambda$ and $h_{\text {rms }}$ values obtained from AFM measurements, we obtain $\tau_{y}^{(1)} / \tau_{x}^{(1)} \approx 10$ for $n=10^{12} \mathrm{~cm}^{-2}$. This ratio leads to $\Delta \rho\left(70^{\circ}\right) / \Delta \rho\left(20^{\circ}\right) \approx 0.2$ in good agreement with the experiment ${ }^{9}$.

\section{CONCLUSIONS AND OUTLOOK}

In this paper we studied the effect of random magnetic fields on the transport properties of a rippled graphene flake. We used the Boltzmann equation, adapted to the case of anisotropic disorder ${ }^{42}$, to address the case of an external magnetic field applied in-plane, the effect of intrinsic strain fields caused by the graphene corrugation, as well as the combination of both.

We find that an external in-plane magnetic field $B_{\|}$ gives rise to very anisotropic conductivity corrections. By neglecting the effect of strain fields and using a parametrization of the ripple disorder that is consistent with experiments, we find conductivity corrections that scale with $B_{\|}^{2}$ and $|n|^{-2}$, consistent with Ref. 9. In contrast, we obtain $\tau_{y}^{(1)} / \tau_{x}^{(1)}$ ratios as large as $20 \cdots 30$.

In the absence of an external magnetic field, random gauge fields due to ripple disorder give a isotropic contribution to the electron momentum relaxation in graphene, in line with the order of magnitude estimate presented in Ref. 30. We find, however, that ripple disorder cannot be neglected in the analysis of the conductivity in the presence of a large $B_{\|}$. We also conclude that, due to the anisotropic nature of the problem, Matthiessen's rule is not accurate to address both intrinsic and extrinsic random fields at the same footing. For that purpose we have to invert the total $M$-matrix.

This approach allows us to successfully describe the corrections to the Drude conductivity reported in the experiment ${ }^{9}$ using typical $\lambda$ and $h_{\text {rms }}$ parameters taken from the AMF literature. In addition, we also obtain a suppression of the resistivity anisotropy (with respect to the case where strain is neglected) that consistent with Ref. 9. We believe that the anisotropic nature of the random magnetic field disorder also significantly changes the quantum corrections to the conductivity with respect to the isotropic result ${ }^{28}$ and deserves further investigation.

In summary, our results suggest that the investigation of anisotropy corrections to the Drude conductivity can be a new and insightful path to experimentally quantify effects of random pseudo-magnetic fields due to strain.

\section{ACKNOWLEDGMENTS}

We thank Eduardo Mucciolo for numerous discussions. This work has been supported by the Brazilian funding agencies CAPES, CNPq, and FAPERJ. 
1 A. H. Castro Neto, F. Guinea, N. M. R. Peres, K. S. Novoselov, and A. K. Geim, Rev. Mod. Phys. 81, 109 (2009).

2 E. R. Mucciolo and C. H. Lewenkopf, J. Phys. Condens. Matter 22, 273201 (2010).

3 N. M. R. Peres, Rev. Mod. Phys. 82, 2673 (2010).

4 S. Das Sarma, S. Adam, E. H. Hwang, and E. Rossi, Rev. Mod. Phys. 83, 407 (2011).

5 A. K. Geim and K. S. Novoselov, Nat. Mater. 6, 183 (2007).

${ }^{6}$ C. H. Lewenkopf, E. R. Mucciolo, and A. H. Castro Neto, Phys. Rev. B 77, 081410(R) (2008).

7 C. H. Lewenkopf and E. R. Mucciolo, J. Comput. Electron. 12, 203 (2013).

8 P. M. Ostrovsky, I. V. Gornyi, and A. D. Mirlin, Phys. Rev. B 74, 235443 (2006).

9 M. B. Lundeberg and J. A. Folk, Phys. Rev. Lett. 105, 146804 (2010).

10 S. Wada, N. Okuda, and J. Wakabayashi, Phys. E 42, 1138 (2010)

11 J. Wakabayashi and K. Sano, J. Phys. Soc. Jpn. 81, 13702 (2012).

12 N. J. G. Couto, D. Costanzo, S. Engels, D.-K. Ki, K. Watanabe, T. Taniguchi, C. Stampfer, F. Guinea, and A. F. Morpurgo, Phys. Rev. X 4, 041019 (2014).

13 M. Ishigami, J. H. Chen, W. G. Cullen, M. S. Fuhrer, and E. D. Williams, Nano Lett. 7, 1643 (2007).

14 V. Geringer, M. Liebmann, T. Echtermeyer, S. Runte, M. Schmidt, R. Rückamp, M. C. Lemme, and M. Morgenstern, Phys. Rev. Lett. 102, 076102 (2009).

15 A. Deshpande, W. Bao, F. Miao, C. N. Lau, and B. J. LeRoy, Phys. Rev. B 79, 205411 (2009).

16 W. G. Cullen, M. Yamamoto, K. M. Burson, J. H. Chen, C. Jang, L. Li, M. S. Fuhrer, and E. D. Williams, Phys. Rev. Lett. 105, 215504 (2010).

17 E.-A. Kim and A. H. Castro Neto, EPL (Europhysics Lett. 84, 57007 (2008).

18 F. de Juan, M. Sturla, and M. A. H. Vozmediano, Phys. Rev. Lett. 108, 227205 (2012).

19 F. Guinea, B. Horovitz, and P. Le Doussal, Phys. Rev. B 77, 205421 (2008).

20 A. Cortijo, F. Guinea, and M. A. H. Vozmediano, J. Phys. A 45, 383001 (2012).

21 M. A. H. Vozmediano, M. I. Katsnelson, and F. Guinea, Phys. Rep. 496, 109 (2010).

22 N. Levy, S. A. Burke, K. L. Meaker, M. Panlasigui, A. Zettl, F. Guinea, A. H. Castro Neto, and M. F. Crommie, Science (80-. ). 329, 544 (2010).
23 P. M. Mensz and R. G. Wheeler, Phys. Rev. B 35, 2844 (1987).

24 W. R. Anderson, D. R. Lombardi, R. G. Wheeler, and T.-P. Ma, IEEE Electron Device Lett. 14, 351 (1993).

${ }^{25}$ D. M. Zumbühl, J. B. Miller, C. M. Marcus, V. I. Fal'ko, T. Jungwirth, and J. S. Harris Jr., Phys. Rev. B 69, 121305 (2004).

26 E. McCann, K. Kechedzhi, V. I. Fal'ko, H. Suzuura, T. Ando, and B. L. Altshuler, Phys. Rev. Lett. 97, 146805 (2006).

27 K. Kechedzhi, E. McCann, V. I. Fal'ko, H. Suzuura, T. Ando, and B. L. Altshuler, Eur. Phys. J. Spec. Top. 148, 39 (2007).

28 H. Mathur and H. U. Baranger, Phys. Rev. B 64, 235325 (2001).

29 D. S. Novikov, Appl. Phys. Lett. 91, 102102 (2007).

30 M. I. Katsnelson and A. K. Geim, Phil. Trans. R. Soc. A 366, 195 (2008).

31 H. Suzuura and T. Ando, Phys. Rev. B 65, 235412 (2002).

32 J. L. Mañes, Phys. Rev. B 76, 045430 (2007).

33 Since we only considered the out of plane contribution in the strain tensor, Eq. (22) gives a slightly different correlation function than the one found in Ref. 19, where a more general expression for the strain tensor was used.

34 A. P. Hinz, S. Kettemann, and E. R. Mucciolo, Phys. Rev. B 89, 075411 (2014).

35 J. W. Kłos and I. V. Zozoulenko, Phys. Rev. B 82, 081414 (2010)

36 C. Kittel, Quantum Theory of Solids, 2nd ed. (Wiley, New York, 1987).

37 P. Hedegard and A. Smith, Phys. Rev. B 51, 10869 (1995).

38 R. R. Brandão and L. Moriconi, arXiv:1410.6706 (2014).

39 J. M. Ziman, Principles of the Theory of Solids (Cambridge University Press, Cambridge, 1972).

40 C. Herring and E. Vogt, Phys. Rev. 101, 944 (1956).

41 E. H. Sondheimer, Proc. R. Soc. Lond. A 268, 100 (1962).

42 Y. Tokura, Phys. Rev. B 58, 7151 (1998).

43 Microreversibility is usually invoked to guarantee $\mathcal{W}\left(\theta, \theta^{\prime}\right)=\mathcal{W}\left(\theta^{\prime}, \theta\right)$ and Eq. (24). In the presence of an external magnetic field, that breaks time-reversal symmetry, $\mathcal{W}\left(\theta, \theta^{\prime}\right)=\mathcal{W}\left(\theta^{\prime}, \theta\right)$ is still true within the approximation used in Eq. (25).

${ }^{44}$ K. Genma and M. Katori, arXiv:1211.2046 (2012).

45 A. W. Rushforth, B. L. Gallagher, P. C. Main, A. C. Neumann, M. Henini, C. H. Marrows, and B. J. Hickey, Phys. Rev. B 70, 193313 (2004). 